\title{
Evaluation criteria of landslide stability
}

\author{
Vladimir Simonyan ${ }^{1, *}$, and Alexander Labuznov ${ }^{1}$ \\ ${ }^{1}$ Moscow State University of Civil Engineering, Yaroslavskoe shosse, 26, Moscow, 129337, Russia
}

\begin{abstract}
The theoretical issues related to the definition of the landslide movement for rectilinear and circular cylindrical sliding surfaces are considered. Based on the concepts of the theory of measurement errors, an analysis of the impact of the parameters on the landslide velocity is performed. The formulas obtained allow us to calculate the speeds of landslides during seismic and atmospheric action.
\end{abstract}

\section{Introduction}

The study of landslide processes has always been and will remain an extremely relevant problem in business activities and, particularly, in the field of construction operation. Due to possible landslide movements on the slopes, there is a danger of disrupting the stability of buildings and structures with the result that they lose the ability to fulfill their natural functions. The Ministry of Emergency situations of Russia annually registers the landslides that have already taken place and predicts possible symptoms of landslides throughout our country. It is no accident, therefore, that landslide processes are the subject of comprehensive theoretical research [1-8]. Geotechnical scientists have thoroughly studied such issues as the physical and mechanical properties of soil rocks, pressure distribution in the landslide body from external influences and the stability of slopes. However, there are still many questions to study landslide processes.

\section{Materials and Methods}

In [9], it has been suggested to calculate the potential possibility of the momentum of a landslide by the formula:

$$
\sin v-\cos v \operatorname{tg} \varphi-\frac{c S}{m g}>0
$$

wherein $v$ is the steepness of slope;

$\varphi$ is the angle of internal friction, the tangent of which is equal to the coefficient of internal friction of the soil;

$c$ is the specific cohesion;

$S$ is the area of a landslide body;

$m$ is the mass of a landslide body;

$g$ is acceleration of gravity, equal to 9.8 meters per seconds squared.

\footnotetext{
* Corresponding author: simonyan.vladimir55@gmail.com
} 
If (1) is less than zero, then the landslide movement is unlikely.

It is clear from (1) that the mobility of landslide depends on the slope steepness $v$, the angle of internal friction $\varphi$, the specific cohesion $c$, the slope area $S$ and the landslide mass $m$, which in turn depends on the depth of sliding surface and the soil composition.

For example, consider cases in which the parameters $\varphi$ and $c$ are variables on the sliding surface, which depend on $x$ (the beginning of break line). Then we need to analyze the formula (1) for different $c$ and $\varphi$ along the entire length $L$ of the sliding line.

Suppose that $\varphi$ and $c$ depend on $x$. For example, consider the case in which these values are constant at certain intervals that are, generally speaking, different. Let $\varphi$ be a constant on $k$ segments ( $K$ partition), with a constant on $n$ segments ( $N$ partition). Then the inequality will be viewed as

$$
m g\left(\sin v-\cos v \sum_{i=1}^{i=k} \operatorname{tg} \varphi_{i}\right)-S \sum_{j=1}^{j=n} c_{j}>0 .
$$

Take a sum of $K$ and $N$ partitions as the $M$ partition of the segment $L$. Then the inequality can be written as

$$
m g\left(\sin v-\cos v \sum_{i=1}^{i=m} \operatorname{tg} \varphi_{i}\right)-S \sum_{i=1}^{i=m} c_{i}>0
$$

The case when for each $c_{j}$ the condition

$$
m g\left(\sin v-\cos v \operatorname{tg} \varphi_{i}\right)-S c_{i}>0,
$$

has been met is trivial and does not require a specific analysis.

Suppose that this inequality is not fulfilled at some (one or more) of the intervals of $i_{t}$ because of the significant $c_{t}$ value. Then the condition of the landslide movement will be

$$
m g\left(\sin v-\cos v \sum_{i=1}^{i=m} \operatorname{tg} \varphi_{i}\right)-S \sum_{i=1}^{i=m} c_{i}>S \sum_{i_{t}} c_{i_{t}},
$$

where on the left side of the inequality (4) the second sum does not include terms with the $i_{t}$ numbers.

Similarly, for $\varphi_{i_{h}}$ at the $i_{h}$ segments, where each $\varphi_{i_{h}}$ is sufficiently small, we will have:

$$
\begin{gathered}
m g\left(\sin v-\cos v \operatorname{tg} \varphi_{h}\right)-S c_{h}<0, \\
-m g\left(\sin v-\cos v \sum_{i_{h}} \operatorname{tg} \varphi_{h}\right)+S \sum_{i_{h}} c_{h}>0, \\
{\left[m g\left(\sin v-\cos v \sum_{i=1}^{i=m} \operatorname{tg} \varphi_{i}\right)-S \sum_{i=1}^{i=m} c_{i}\right]>-m g\left(\sin v-\cos v \sum_{i_{h}} \operatorname{tg} \varphi_{h}\right)+S \sum_{i_{h}} c_{h},}
\end{gathered}
$$

or, which is the same,

$$
\left[m g\left(\sin v-\cos v \sum_{i=1}^{i=m} \operatorname{tg} \varphi_{i}\right)\right]-S \sum_{i=1}^{i=m} c_{i}+m g\left(\sin v-\cos v \sum_{i_{h}} \operatorname{tg} \varphi_{i_{h}}\right)>0,
$$

where amounts into the square brackets do not include terms with $i_{h}$ numbers.

Similarly, consider a landslide with a circular-cylinder sliding surface. Suggest that the landslide body rests on an arc of a circle delimited by lines with angles of inclination $\gamma_{0}$ and $\gamma_{\omega}$. The $\gamma_{0}$ angle of inclination is perpendicular to $v_{0}$, and the angle of inclination is perpendicular to $\boldsymbol{v}_{\omega}$. Divide the arc into $k$ equal intervals so that $\varphi$ and $c$ are constant inside each segment, and replace the small arcs with chords (Figure 1): 


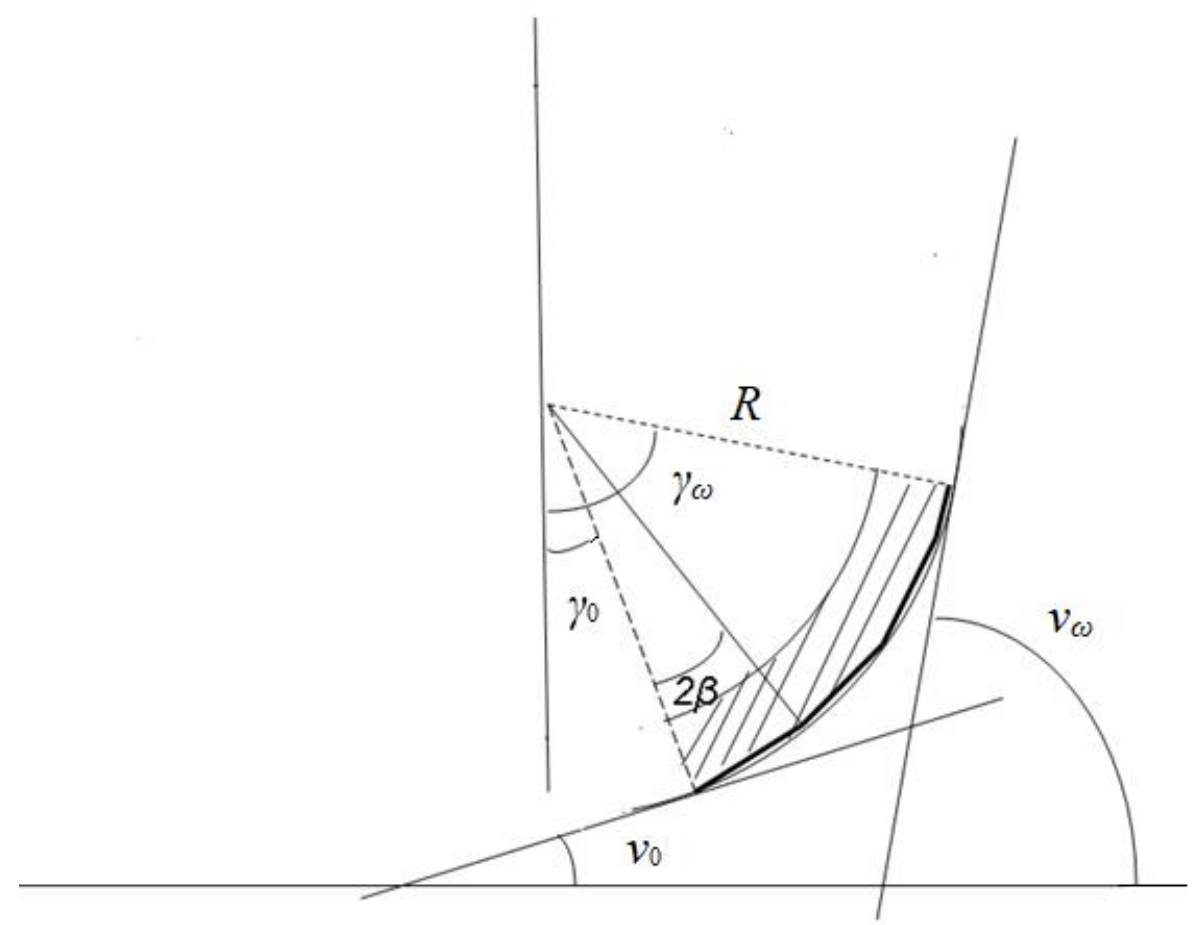

Fig. 1. Division of the arc into k equal intervals.

Then the inequality can be written as

$$
\sum_{i=1}^{i=k} m_{i} g\left(\sin v_{i}-\cos v_{i} \operatorname{tg} \varphi_{i}\right)-\sum_{i=1}^{i=k} S_{i} c_{i}>0
$$

where $S_{i}=2 R a \sin \beta$;

$a$ is the landslide width;

$R$ is the radius of the slip curve;

$\beta=\frac{\left(\gamma_{\omega}-\gamma_{0}\right)}{2 k}$.

Then

$$
\begin{gathered}
\sum_{i=1}^{i=k} m_{i} g\left(\sin v_{i}-\cos v_{i} \operatorname{tg} \varphi_{i}\right)-\sum_{i=k}^{i=k} 2 R a \sin \beta c_{i}>0, \\
g \sum_{i=1}^{i=k} m_{i}\left(\sin v_{i}-\cos v_{i} \operatorname{tg} \varphi_{i}\right)-2 R a \sin \beta \sum_{i=1}^{i=k} c_{i}>0 .
\end{gathered}
$$

Since $m_{i}=\rho h^{\prime} S_{i}$, wherein $h^{\prime}$ is constant if the depth of location of the sliding surface of a landslide is constant. Then:

$$
h^{\prime} S g \sum_{i=1}^{i=k} \rho_{i} \sin v_{i}-\sum_{i=1}^{i=k} \rho_{i} \cos v_{i} \operatorname{tg} \varphi_{i}-2 R a \sin \beta \sum_{i=1}^{i=k} c_{i}>0 .
$$

Thus, according to formulas (8) and (12), it is possible to calculate the likelihood of landslide movement for rectilinear and circular-cylinder surfaces of the landslide "bed", respectively. 


\section{Results}

From (1), the landslide velocity $V$ can be found from the expression

$$
V=t\left(g \cdot \sin v-g \cdot \cos v \cdot \operatorname{tg} \varphi-\frac{c S}{m}\right) .
$$

Consider the following example. Suppose that: $v=25^{\circ} ; \varphi=14^{\circ} ; c=37 \mathrm{kPa} ; S=10$ ha; $h=10 \mathrm{~m} ; \rho=1,91$ tons per cubic metre. Then we will have:

$$
V=\left(9,8 \frac{\mathrm{m}}{\mathrm{sec}^{2}} \sin 25^{\circ}-9,8 \frac{\mathrm{m}}{\mathrm{sec}^{2}} \cos 25^{\circ} \operatorname{tg} 14^{\circ}-\frac{37 \frac{\mathrm{ton}}{\mathrm{m} \cdot \mathrm{sec}^{2}} \cdot 100000 \mathrm{~m}^{2}}{1910000 \mathrm{ton}}\right) \mathrm{sec}=-0,01 \frac{\mathrm{m}}{\mathrm{sec}} .
$$

Analyze the result. The symbol "-" indicates that the landslide stands still. However, the result close to zero means that the landslide is in a stress-strain state and when one of the factors affecting the landslide works the landslide will move.

The parameters $v, \varphi, c, S, m$ are determined empirically and are of stochastic nature, imposing some uncertainty on the accounting result by formula (13). Analyze the impact of the parameters entering formula (13) on the landslide velocity based on the concepts of the theory of measurement errors. Dispersion (13) is equal to:

$$
\sigma_{V}^{2}=\left(\frac{\partial V}{\partial v} \sigma_{v}\right)^{2}+\left(\frac{\partial V}{\partial \varphi} \sigma_{\varphi}\right)^{2}+\left(\frac{\partial V}{\partial c} \sigma_{c}\right)^{2}+\left(\frac{\partial V}{\partial S} \sigma_{S}\right)^{2}+\left(\frac{\partial V}{\partial m} \sigma_{m}\right)^{2} .
$$

By taking the partial derivatives of this function, we obtain the coefficients for all five arguments:

$$
\begin{aligned}
& \quad \frac{\partial V}{\partial v}=t \cdot g(\cos v+\sin v \operatorname{tg} \varphi) ; \quad \frac{\partial V}{\partial \varphi}=t \cdot g \cdot \cos v\left(\frac{1}{\cos ^{2} \varphi}\right) ; \quad \frac{\partial V}{\partial c}=t \frac{S}{m} ; \quad \frac{\partial V}{\partial S}=t \frac{c}{m} ; \\
& \frac{\partial V}{\partial m}=-t \frac{c S}{m^{2}} .
\end{aligned}
$$

Turning to the standard uncertainties we will have:

$$
m_{V}^{2}=t^{2}\left(\begin{array}{l}
g^{2} \cos ^{2} v \frac{m_{v}^{2}}{\rho^{2}}+g^{2} \sin ^{2} v \cdot \operatorname{tg}^{2} \varphi \frac{m_{v}^{2}}{\rho^{2}}+g^{2} \cos ^{2} v\left(\frac{1}{\cos ^{2} \varphi}\right)^{2} \frac{m_{\varphi}^{2}}{\rho^{2}}+ \\
+\frac{S^{2}}{m^{2}} m_{c}^{2}+\frac{c^{2}}{m^{2}} m_{S}^{2}+\frac{c^{2} S^{2}}{\left(m^{2}\right)^{2}} m_{m}^{2}
\end{array}\right)
$$

Within the values given above we obtain the following coefficients of an equation (15):

$$
m_{V}^{2}=t^{2}\left(0,024 m_{v}^{2}+0,027 m_{\varphi}^{2}+0,0027 m_{c}^{2}+3,8 \cdot 10^{-10} m_{S}^{2}+1,0 \cdot 10^{-12} m_{m}^{2}\right) .
$$

Calculate the sum of the coefficients of equation (16). We obtain: $\Sigma=0,054$. Take this sum for $100 \%$ and find the percentage of each coefficient. We get:

$$
\begin{array}{ll}
- & \frac{0,024}{0,054} \cdot 100 \%=45 \% ; \\
- & \frac{0,027}{0,054} \cdot 100 \%=50 \% ; \\
- & \frac{0,0027}{0,054} \cdot 100 \%=5 \%
\end{array}
$$

Thus, the landslide velocity $V$ is mainly influenced by the slope steepness $v$, the angle of internal friction $\varphi$, and the specific cohesion $c$. 
Consider a situation in which an earthquake provokes the activation of landslide processes.

Consideration of the earthquake action includes two methods:

- fictitious slope;

- fictitious seismic angle.

The core of the fictitious slope method [10] is that a slope can turn at the angle $\Delta v$ at which the resultant of gravitational and horizontally directed earthquake forces is inclined away from the vertical. The action of the earthquake is modeled by the short-term inclination of the slope at an angle, the value of which, depending on the seismic coefficient $\varepsilon$, is given in Table 1 [11].

Table 1. Increase in the angle of slope depending on the intensity of earthquakes.

\begin{tabular}{|c|c|c|c|c|}
\hline $\begin{array}{c}\text { Seismicity coefficient } \varepsilon \text { (in fractions of } \\
\text { the acceleration of gravity } g \text { ) }\end{array}$ & 0.05 & 0.1 & 0.2 & 0.4 \\
\hline $\begin{array}{c}\text { Increase in the angle of slope } \Delta v \text { in } \\
\text { degrees }\end{array}$ & 3 & 6 & 12 & 24 \\
\hline
\end{tabular}

With respect to formula (13), we obtain

$$
V=t\left[g \sin (v+\Delta v)-g \cos (v+\Delta v) \operatorname{tg} \varphi-\frac{c S}{m}\right] .
$$

With the same data and $\varepsilon=0,2, \Delta v=12^{\circ}$, we obtain the velocity $V=2 \mathrm{~m} / \mathrm{sec}$.

The essence of the method of the seismic angle is that the action of the earth-wake is modeled by reducing the angle of internal friction of the soils of the main deformed horizons by seismic magnitude and by changing the value of the normal pressure. The seismic magnitude as a function of the value of earthquake acceleration is given in Table 2.

Table 2. The values of calculated magnitudes of earthquake acceleration and the seismic angle.

\begin{tabular}{|c|c|c|c|c|}
\hline $\begin{array}{c}\text { Seismicity coefficient } \varepsilon \text { (in fractions of } \\
\text { the acceleration of gravity } g \text { ) }\end{array}$ & 0.05 & 0.1 & 0.2 & 0.4 \\
\hline Seismic angle $\delta$ in degrees & 3 & 6 & 12 & 24 \\
\hline
\end{tabular}

With respect to formula (13), we obtain

$$
V=t\left[g \sin v-g \cos v \operatorname{tg}(\varphi-\delta)-\frac{c S}{m(1+\varepsilon)}\right] .
$$

With the same data and $\varepsilon=0,2, \delta=12^{\circ}$, we obtain the velocity $V=2 \mathrm{~m} / \mathrm{sec}$.

The impact of groundwaters on the state of the landslide slope is reflected in various ways, by changing the stress state of the massif and physical and mechanical properties of the soils, as well as by causing the development of filtration deformations and, in particular, shear strength, reducing the value of shearing resistance [12]. At the same time, water, due to the emergence of pore pressure, changes the magnitude of stress having an impact on the soil skeleton.

As is known, the impact of pore pressure on the shear resistance is described by the equation:

where $u$ is the pore pressure.

$$
\tau=(\sigma-u) \operatorname{tg} \varphi+c,
$$

Consider a situation in which the pore pressure is maximum, i.e. $u=\sigma$.

Then

$$
V=t\left(g \sin v-\frac{c S}{m}\right) .
$$

With the same data, we obtain the velocity $V=2 \mathrm{~m} / \mathrm{sec}$. 


\section{Conclusions}

That means that if the landslide is in a stress-strain state, then an earthquake with the magnitude $\mathrm{M}=8$ provokes the same rate of the landslide movement, as well as heavy rain, which increases the level of groundwaters. Accordingly, at a smaller magnitude (less precipitation), the rate of landslide movement decreases. At $M=6$, the rate of landslide movement will be "very rapid" with the transition to "exceptionally rapid".

\section{References}

1. E.V. Bezuglova, Mathematical determination of the stability reserve of landslide objects (Krasnodar, 2014)

2. A.S. Bobrovich, Mathematical determination of the stability reserve of landslide objects (Moscow, 2008)

3. A.I. Kuznecov, Development of a method for determining the slip surface of a landslide according to geodetic monitoring data (Moscow, 2012)

4. O.G. Pavlovskaya, Analysis and assessment of the geodetic data of the dynamics of landslides in conditions of blasting and unloading of slopes (Novosibirsk, 2012)

5. I.K. Fomenko, Engineering geology, 6 (2012)

6. V.M. Kutepova, A.I. Sheko, Exogenous geological hazards. Theme volume (Moscow, KRUK, 2002)

7. V.V. Simonyan, Substantiation of accuracy and development of methods for mathematical and statistical analysis of geodetic observations of landslide displacement (Moscow, 2008)

8. V.V. Simonyan, Study of landslide processes by geodetic methods (Moscow, Izd-vo Moskovskogo stroit. Universiteta, 2015)

9. V.V. Simonyan, A.G. Tamrazyan, Vestnik MGSU, 7 (2016)

10. E.V. Kalinin, Engineering-geological calculations and modeling (Moscow, Izd. Moskovskogo universiteta, 2006)

11. M.H. Zyong, I.K. Fomenko, V.V. Pendin, Engineering geology, 1 (2013)

12. L.K. Ginzburg, Recommendations on the choice of methods for calculating the coefficient of slope stability and landslide pressure (Moscow, Central'noe byuro nauchno-tekhnicheskoj informacii, 1986) 